\title{
A importância da fisioterapia em pacientes pré e pós-operatório de cirurgia de revascularização do miocárdio
}

The importance of physiotherapy in pre and post-operative myocardial arterial surgery patients La importancia de la fisioterapia en pacientes pre y postoperatorios de cirugía arterial miocárdica

Lenara Almeida de Moraes ORCID: https://orcid.org/0000-0003-1136-8137 Faculdade de Ensino Superior do Piauí, Brasil E-mail: lenaraa92@gmail.com

Kelma Regina Galeno Pinheiro ORCID: https://orcid.org/0000-0003-1925-7079 Faculdade de Ensino Superior do Piaú, Brasil E-mail: kelmagaleno@yahoo.com.br

Diane Nogueira Paranhos Amorim ORCID: https://orcid.org/0000-0002-5362-5747 Faculdade de Ensino Superior do Piauí, Brasil E-mail: dnpa.fisio@gmail.com

Ruth Raquel Soares de Farias ORCID: https://orcid.org/0000-0002-0988-0900 Faculdade de Ensino Superior do Piauí, Brasil E-mail: ruthraquelsf@gmail.com

José Edmilson Da Silva Neto ORCID: https://orcid.org/0000-0002-6372-0481 Faculdade de Ensino Superior do Piauí, Brasil E-mail: edmilson161214@gmail.com

Ester Miranda de Sousa ORCID: https://orcid.org/0000-0003-2319-4840 Faculdade de Ensino Superior do Piauí, Brasil E-mail: mirandaester70@gmail.com

Luigi Gabriel Brasil da Silva ORCID: https://orcid.org/0000-0002-8556-3958 Faculdade de Ensino Superior do Piaú, Brasil E-mail: luigigabrie1010@ outlook.com

Israel Wesley Pinto Leal ORCID: https://orcid.org/0000-0001-5394-6623 Faculdade de Ensino Superior do Piaú, Brasil

E-mail: israelleal373@gmail.com

Francisco Maurílio Da Silva Carrias ORCID: https://orcid.org/0000-0002-0762-0494 Faculdade de Ensino Superior do Piaú, Brasil E-mail: mauriliocarrias@gmail.com

Hengrid Graciely Nascimento Silva ORCID: https://orcid.org/0000-0002-7362-0118 Faculdade de Ensino Superior do Piauí, Brasil

E-mail: hengrid_graciely@hotmail.com

Ivisson Lucas Campos da Silva ORCID: https://orcid.org/0000-0003-2848-9402 Faculdade de Ensino Superior do Piauí, Brasil E-mail: ivissonlucas@hotmail.com

Lélia Lilianna Borges de Sousa Macedo ORCID: https://orcid.org/0000-0003-1108-6324 Faculdade de Ensino Superior do Piauí, Brasil E-mail: leliafisio@hotmail.com

Thais Cristina da Costa Rocha Pereira ORCID: https://orcid.org/0000-0002-2020-6722 Faculdade de Ensino Superior do Piauí, Brasil E-mail: thaisinha.ibr@gmail.com

\section{Resumo}

As cirurgias cardíacas são procedimentos graves que, embora considerados seguros, são acompanhados por processos complexos. Nesse contexto, a fisioterapia é parte integrante na gestão do atendimento ao paciente cardíaco, tanto no pré-operatório como no pós-operatório, pois contribui significativamente para um melhor prognóstico do paciente que 
irá ou já realizou a CRM. Objetivo foi descrever os efeitos da fisioterapia na reabilitação de pacientes no pré e póscirúrgico de Revascularização do Miocárdio. Trata- se de uma pesquisa sistemática por meio da busca de artigos nas bases de dados da SciELO, PubMed/ MEDLINE e BIREME. Foram encontrados 104 artigos nas bases de dados pesquisadas, destes, nove foram incluídos para compor a discussão. Os estudos evidenciaram que as técnicas de fisioterapia são muito importantes e que, por meio de atividade física aeróbica, contribuem para que não ocorram complicações na recuperação, diminui o quadro álgico e melhora a qualidade de vida, apresentando eficácia também em outros eventos cardiovasculares. Como consideração final, o estudo mostrou que a fisioterapia pré-operatória provou ter ferramentas que podem auxiliar os fisioterapeutas na reabilitação de pacientes, facilitando sua recuperação e que deve ser uma prática adaptada às características da população atendida.

Palavras-chave: Cirurgia cardíaca; Revascularização do miocárdio; Fisioterapia; Pré-operatório; Pós-operatório; Qualidade de vida.

\begin{abstract}
Cardiac surgeries are serious procedures that, although considered safe, are accompanied by complex processes. In this context, respiratory physiotherapy is an integral part of cardiac patient care management, both preoperatively and postoperatively, as it significantly contributes to a better prognosis. in the case of a patient who is going to or has already undergone CABG surgery, physiotherapy becomes important, as it contributes to a better patient's recovery. Objective was to describe the effects of physical therapy on the rehabilitation of patients before and after myocardial revascularization surgery. This is a systematic search through the search for articles in the SciELO, PubMed/MEDLINE and BIREME databases. One hundred and four (104) articles were found in the searched databases; of these, nine (9) were included to compose the discussion. Studies have shown that respiratory physiotherapy techniques are very important and that, through aerobic physical activities, it contributes to avoid complications in recovery, reduces pain and improves quality of life, also showing effectiveness in other cardiovascular events. As a final consideration, the study showed that preoperative physical therapy proved to be a tool that can help physical therapists in the rehabilitation of patients, facilitating their recovery and that it should be a practice adapted to the characteristics of the population served.
\end{abstract}

Keywords: Cardiac surgery; Revascularization of the myocardium; Physiotherapy; Preoperative; Postoperative; Quality of life.

\title{
Resumen
}

Las cirugías cardíacas son procedimientos serios que, aunque se consideran seguros, van acompañados de procesos complejos. En este contexto, la fisioterapia respiratoria es una parte integral del manejo de la atención al paciente cardíaco, tanto preoperatoria como posoperatoriamente, ya que contribuye significativamente a un mejor pronóstico. en el caso de un paciente que va o ya ha sido sometido a una cirugía de CABG, la fisioterapia cobra importancia, ya que contribuye a una mejor recuperación del paciente. El objetivo fue describir los efectos de la fisioterapia en la rehabilitación de pacientes antes y después de la cirugía de revascularización miocárdica. Se trata de una búsqueda sistemática a través de la búsqueda de artículos en las bases de datos SciELO, PubMed / MEDLINE y BIREME. Se encontraron ciento cuatro (104) artículos en las bases de datos buscadas; de estos, nueve (9) fueron incluidos para componer la discusión. Los estudios han demostrado que las técnicas de fisioterapia respiratoria son muy importantes y que, a través de la actividad física aeróbica, contribuye a evitar complicaciones en la recuperación, reduce el dolor y mejora la calidad de vida, mostrando también efectividad en otros eventos cardiovasculares. Como consideración final, el estudio mostró que la fisioterapia preoperatoria resultó ser una herramienta que puede ayudar a los fisioterapeutas en la rehabilitación de los pacientes, facilitando su recuperación y que debe ser una práctica adaptada a las características de la población atendida.

Palabras clave: Cirugía cardíaca; Revascularização del miocardio; Fisioterapia; Preoperatorio; Postoperatorio; Calidad de vida.

\section{Introdução}

As doenças cardiovasculares estão entre as principais causas de mortes em países desenvolvidos e sua ocorrência escalou epidemicamente em países em desenvolvimento. Uma das alternativas para tratamento da doença arterial coronariana, é a Revascularização do Miocárdio (CRM), que contribui para se ter uma qualidade de vida a longo prazo, sem precisar de procedimento com frequência (Cavenaghi et al., 2011).

As cirurgias cardíacas são procedimentos graves que, embora considerados seguros, são acompanhados por processos complexos como anestesia geral, circulação extracorpórea, ventilação mecânica e repouso no leito. Além desses fatores, os pacientes cardíacos apresentam função prejudicada como resultado da redução da capacidade oxidativa do músculo esquelético e da perfusão muscular diminuída precisando ter que ir para a ventilação mecânica (VM) (Araújo, et al., 2016). 
Nesse contexto, a fisioterapia é parte integrante da gestão do atendimento ao paciente cardíaco, tanto no préoperatório como no pós-operatório, pois colabora expressivamente para melhor prognóstico desses pacientes por meio de técnicas específicas. Técnicas-de higiene brônquica, reexpansão e aspiração, promovem benefícios, tais como a melhora da oxigenação, melhora da complacência pulmonar, do volume corrente expiratório e da remoção de secreção brônquica (Costa et al., 2020).

No pré-operatório, pode contribuir no preparo do paciente que será submetido à revascularização cirúrgica do músculo cardíaco, melhorando sua eficiência respiratória, com o uso de técnicas como espirometria motivacional, exercícios de respiração profunda, treinamento muscular inspiratório, e na educação em saúde ao paciente e familiar ou cuidadores, informando sobre os cuidados necessários durante todo o processo (Sá et al., 2020).

No pós-operatório, o objetivo é melhorar a capacidade respiratória quando há uma complicação pulmonar instalado, realizada por meio de manobras fisioterapêuticas e dispositivos de respiração não invasivos projetados para melhorar mecânica respiratória, reexpansão pulmonar e higiene brônquica. Nesse caso, os benefícios contribuem para a alta hospitalar, reabilitação precoce e retorno às atividades diárias (Leguisamo et al., 2005). A fisioterapia além desses benefícios, também auxiliar na reintegração do paciente ao meio social, reduzindo os efeitos negativos ou prejudiciais à saúde que podem estar associados ao confinamento do leito, melhorando sua qualidade de vida (Santos et al., 2019).

Logo, no caso do paciente que irá ou já realizou a CRM, a fisioterapia se torna importante, pois contribui para uma melhor recuperação do paciente. A CRM é um procedimento recomendado para grupos selecionados de pacientes com estreitamento grave ou "bloqueio" das artérias que irrigam o coração. A operação visa melhorar a qualidade de vida dos pacientes, além de aliviar os sintomas causados pelas doenças cardíacas, além de aumentar a sobrevida (Osório et al., 2015).

Assim, o objetivo desse trabalho foi descrever os efeitos da fisioterapia na reabilitação de pacientes no pré e póscirúrgico de Revascularização do Miocárdio. E assim especificamente, elencar as principais intervenções fisioterapêuticas realizadas no pré e pós-operatório de pacientes cardiopatas submetidos a CRM, como também destacar a importância da inclusão do profissional fisioterapeuta nas equipes hospitalares durante o processo de pré e pós-cirúrgico com pacientes cardíacos.

\section{Metodologia}

Trata- se de uma revisão sistemática, que buscou reunir amostras de estudos científicos respectivos à intervenção da fisioterapia no pré e pós-operatório de CRM, sendo realizada por meio da busca de artigos nas bases de dados da Scientific Electronic Library Online (SciELO), PubMed/ MEDLINE e Centro Latino-Americano e do Caribe de Informação em Ciências da Saúde (BIREME).

A coleta de dados foi realizada entre agosto/setembro de 2021, utilizando os seguintes descritores e suas combinações: "doenças cardiovasculares", "revascularização do miocárdio", "fisioterapia cardiovascular", "pré-operatório" e "pósoperatório". Os descritores foram consultados no DeCS.

Ao final da busca nas bases de dados, foram realizadas revisões dos artigos pelo título e resumo verificando se os mesmos contemplam os critérios de inclusão sendo eles: artigos com pacientes submetidos à cirurgia de revascularização do miocárdio, entre os anos de 2015 e 2021, ensaios clínicos randomizados e não randomizados, estudo transversal e estudo de caso controle. Foram excluídas publicações que não incluíam pessoas pós-processo de CRM, que tratavam de pacientes com cardiopatias congênitas, duplicados e incompletos.

Após a conclusão da avaliação das publicações selecionadas, foram apresentados em forma de tabela os dados mais importantes como: autor/ano, amostra, intervenção e resultados. 


\section{Resultados}

Foram encontrados 100 artigos nas bases de dados pesquisadas; destes, nove foram incluídos. O fluxograma 1 detalha o processo de inclusão e exclusão dos artigos.

Fluxograma 1. Processo de inclusão e exclusão dos artigos.

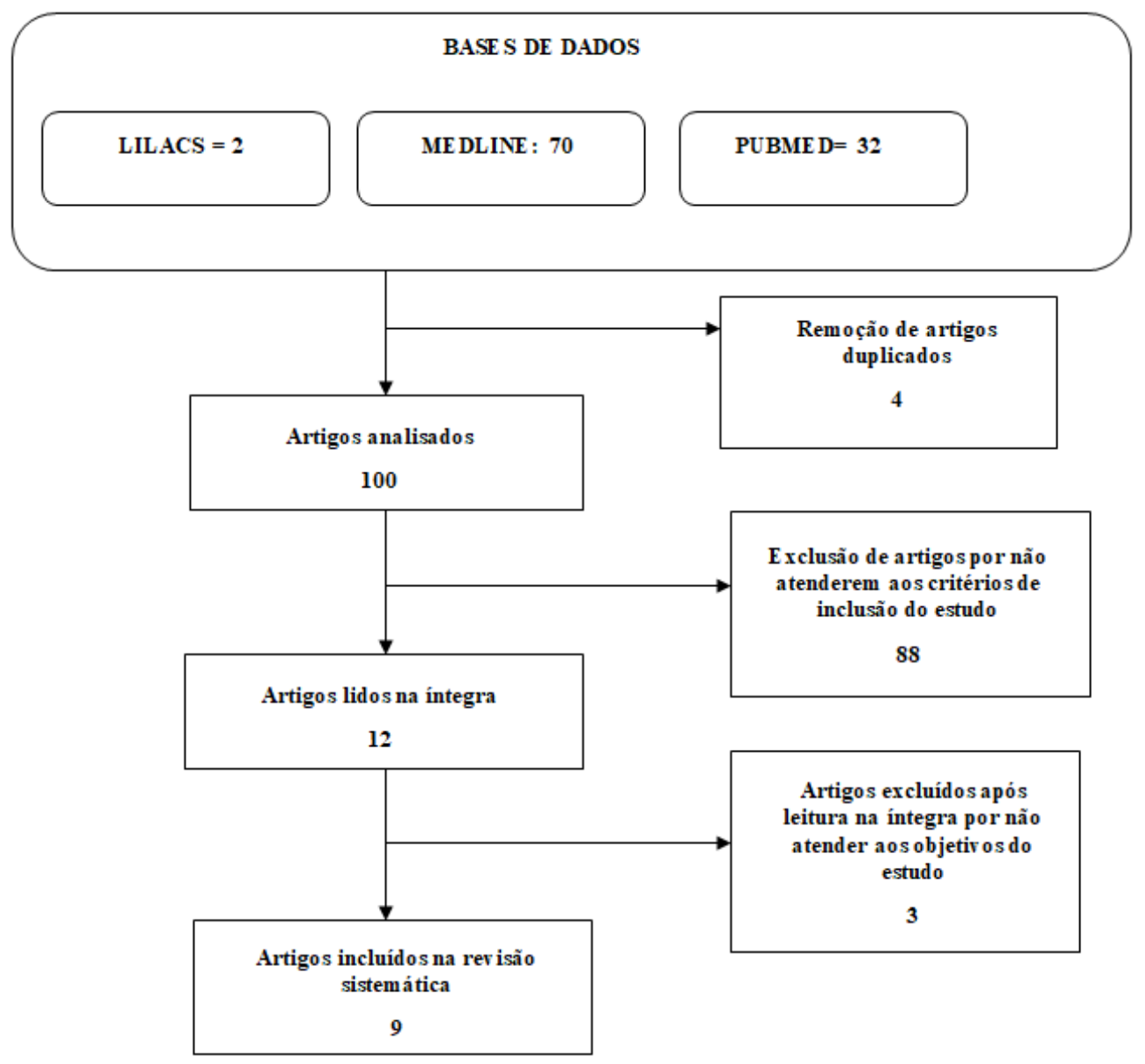

Fonte: Autores.

Após a seleção desses artigos, foram extraídas as seguintes informações: autor, ano de publicação, periódico de publicação, base de dados, objetivo, tipo de pesquisa e evidências encontradas. A análise crítica dos resultados da pesquisa é realizada agrupando os artigos de acordo com a similaridade dos métodos temáticos, conforme mostra no Quadro 1.

Quadro 1 - Distribuição dos estudos quando ao periódico, autores, ano, método e resultados.

\begin{tabular}{|c|c|c|c|}
\hline $\begin{array}{c}\text { Periódico } \\
\text { Autores/ ANO }\end{array}$ & Título & Métodos & Resultados \\
\hline $\begin{array}{l}\text { Polish Archives of } \\
\text { Internal Medicine } \\
\text { Sinnadurai S et al. } \\
\text { (2021) }\end{array}$ & $\begin{array}{l}\text { Effects of cardiac rehabilitation } \\
\text { on risk fator management and } \\
\text { quality of life in patients } \\
\text { with ischemic heart disease: a } \\
\text { multicenter cross-sectional } \\
\text { study. }\end{array}$ & $\begin{array}{l}\text { Comparação entre pacientes que realizaram } \\
\text { reabilitações cardíacas e pacientes que não } \\
\text { realizaram reabilitação cardíaca após } 18 \\
\text { meses da cirurgia de revascularização do } \\
\text { miocárdio. }\end{array}$ & $\begin{array}{l}\text { Pacientes que participaram de um programa } \\
\text { de reabilitação cardíaca tiveram maior } \\
\text { probabilidade de parar de fumar, controlar } \\
\text { os níveis glicêmicos e melhor qualidade de } \\
\text { vida. }\end{array}$ \\
\hline $\begin{array}{l}\text { Fisioterapia Brasil } \\
\text { Cordeiro A et al. } \\
\text { (2021) }\end{array}$ & $\begin{array}{l}\text { Dois tipos de treinamento } \\
\text { muscular inspiratório sobre a } \\
\text { força muscular de pacientes } \\
\text { após revascularização do } \\
\text { miocárdio: ensaio clínico }\end{array}$ & $\begin{array}{l}\text { Os pacientes foram avaliados no pré- } \\
\text { operatório para pressão inspiratória máxima } \\
\left(\mathrm{PI}_{\text {máx }}\right) \text {, pressão expiratória }(\mathrm{PEF}) \text {, pico de } \\
\text { fluxo expiratório (PFE), teste de caminhada } \\
\text { de seis minutos (TC6) e medida de }\end{array}$ & $\begin{array}{l}\text { Em relação às variáveis funcionais, } \\
\text { verificou-se que o grupo TMI no TC 6min } \\
\text { obteve uma distância maior que os demais } \\
\text { grupos. As complicações pulmonares foram } \\
\text { menos evidentes no grupo submetido ao }\end{array}$ \\
\hline
\end{tabular}




\begin{tabular}{|c|c|c|c|}
\hline & & $\begin{array}{l}\text { independência funcional (MIF). Após a } \\
\text { cirurgia, eles foram divididos em três } \\
\text { grupos: grupo controle (GC), grupo } \\
\text { treinamento com carga linear de pressão } \\
\text { (TMI) e grupo incentivo inspiratório (GI). }\end{array}$ & $\begin{array}{l}\text { treinamento muscular inspiratório } \\
\text { convencional quando comparado ao grupo } \\
\text { controle e ao grupo incentivo, com } \\
\text { significância estatística em pneumotórax, } \\
\text { em relação à força e função muscular, } \\
\text { observou-se redução estatisticamente } \\
\text { significativa em todos os grupos, mas no } \\
\text { grupo controle foi observada queda maior } \\
\text { das variáveis durante a internação. }\end{array}$ \\
\hline $\begin{array}{l}\text { Respiratory Care } \\
\text { Windmöller P et al. } \\
\text { (2020) }\end{array}$ & $\begin{array}{l}\text { Exercício físico combinado } \\
\text { com CPAP em indivíduos } \\
\text { submetidos à revascularização } \\
\text { cirúrgica do miocárdio: um } \\
\text { ensaio clínico randomizado }\end{array}$ & $\begin{array}{l}\text { O grupo controle ( } \mathrm{n}=16 \text { sujeitos) iniciou a } \\
\text { reabilitação no pós-operatório imediato com } \\
\text { exercícios respiratórios e mobilização } \\
\text { passiva na posição sentada, evoluindo para } \\
\text { exercícios ativos, deambulação e } \\
\text { treinamento em escada. Para o grupo } \\
\text { intervenção ( } \mathrm{n}=15 \text { sujeitos), exercícios } \\
\text { dinâmicos em cicloergômetro combinados } \\
\text { com CPAP foram adicionados ao programa } \\
\text { de step do segundo ao quarto dia de pós- } \\
\text { operatório em uma única sessão diária. }\end{array}$ & $\begin{array}{l}\text { O tempo de permanência na UTI foi } \\
\text { significativamente menor no grupo de } \\
\text { intervenção, uma redução na capacidade } \\
\text { funcional também foi observada, mas não } \\
\text { foi estatisticamente significativa. Em ambos } \\
\text { os grupos, houve uma diminuição } \\
\text { significativa na } \mathrm{P}_{\operatorname{Imax}} \mathrm{P}_{\mathrm{Emax}} \mathrm{e} \text { teste de } \\
\text { sentar para levantar de } 1 \text { min no } 4^{\circ} \text { dia pós- } \\
\text { operatório em comparação com o pré- } \\
\text { operatório. O melhor desempenho no } \\
\text { testeTC6 ocorreu no grupo intervenção após } \\
\text { o procedimento cirúrgico. }\end{array}$ \\
\hline $\begin{array}{l}\text { Clinical Rehabilitation } \\
\text { Steinmetz C et al. } \\
\text { (2020) }\end{array}$ & $\begin{array}{l}\text { Pré-reabilitação em pacientes } \\
\text { que aguardam cirurgia de } \\
\text { revascularização do miocárdio } \\
\text { eletiva - efeitos na capacidade } \\
\text { funcional e na qualidade de } \\
\text { vida: um ensaio clínico } \\
\text { randomizado }\end{array}$ & $\begin{array}{l}\text { Seleções de pacientes pré-operatórios } \\
\text { eletivos de CRM, foram aleatoriamente } \\
\text { designados para uma intervenção, dividindo- } \\
\text { se em dois grupos, GI: EBPrehab de duas } \\
\text { semanas incluindo exercício aeróbio } \\
\text { supervisionado e GC: cuidados habituais } \\
\text { incluindo teste cardiopulmonar, TC6min, } \\
\text { Timed-Up-and-Go Test (TUG) e QV } \\
\text { (questionário MacNew). }\end{array}$ & $\begin{array}{l}\text { Durante o período pré e pós-operatório, a } \\
\text { distância de caminhada de seis minutos e o } \\
\text { tempo cronometrado melhoraram } \\
\text { significativamente em ambos, como } \\
\text { resultado do período pré-operatório, } \\
\text { interação significativa entre os grupos foi } \\
\text { observada em todos domínios da qualidade } \\
\text { de vida. No GI as melhorias foram mais } \\
\text { pronunciadas em comparação com o GC. }\end{array}$ \\
\hline $\begin{array}{c}\text { Paquistão Journal of } \\
\text { medical Sciences } \\
\text { Sahar W, Ajaz N, Haider } \\
\text { Z, Jalal. } \\
\text { (2020) }\end{array}$ & $\begin{array}{l}\text { Eficácia do treinamento } \\
\text { muscular respiratório pré- } \\
\text { operatório versus tratamento } \\
\text { convencional para melhorar a } \\
\text { saúde pulmonar pós-operatória } \\
\text { após enxerto de bypass da } \\
\text { artéria coronária }\end{array}$ & $\begin{array}{l}\text { Um ensaio clínico prospectivo } \\
\text { randomizado foi realizado com } 60 \text { pacientes } \\
\text { submetidos a CRM. O TC6min foi realizado } \\
\text { como linha de base no G1 e no G2 no dia da } \\
\text { admissão. O oxímetro de pulso portátil de } \\
\text { dedo foi usado para avaliação da saturação } \\
\text { de oxigênio e frequência cardíaca. Em } \\
\text { seguida, os pacientes do G1 receberam } \\
\text { treinamento muscular respiratório (TMR) } \\
\text { que consistia em espirometria de incentivo } \\
\text { (EI), respiração diafragmática, movimento } \\
\text { respiratório segmentar (expansão costal } \\
\text { lateral, expansão costal posterior, exercícios } \\
\text { de expansão costal apical) e huff-tough } \\
\text { técnicas. Um total de } 28 \text { sessões de } \\
\text { treinamento muscular respiratório foi } \\
\text { realizado no G1 por > } 15 \text { minutos em uma } \\
\text { velocidade baixa constante para cada } \\
\text { paciente. Os pacientes do G2 receberam } \\
\text { tratamento convencional que inclui } \\
\text { respiração profunda. }\end{array}$ & $\begin{array}{l}\text { Os pacientes do G1 tiveram um } \\
\text { desempenho melhor do que o } \mathrm{G} 2 \text { em seu } \\
\text { TC6min com valor de } p<0,05 \text {. Da mesma } \\
\text { forma, o G1 apresentou menor tempo de } \\
\text { ventilação mecânica, dependência de } \\
\text { oxigenoterapia e internação pós-operatória } \\
\text { em relação ao G2, apresentando valores de } \\
p<0,05 \text {. }\end{array}$ \\
\hline $\begin{array}{c}\text { Journal of Cardic Surgery } \\
\text { Aquino, T. N., Faria } \\
\text { Rosseto, S., Lúcio Vaz, J., } \\
\text { Faria Cordeiro Alves, C., } \\
\text { Vidigal, F. de C., Galdino, } \\
\text { G. } \\
\text { (2021) }\end{array}$ & $\begin{array}{llr}\text { Avaliação } & \text { do treinamento } \\
\text { muscular } & \text { respiratório e e } \\
\text { periférico em indivíduos } \\
\text { submetidos à revascularização } \\
\text { do miocárdio. }\end{array}$ & $\begin{array}{l}\text { Os } 83 \text { pacientes submetidos a CRM foram } \\
\text { divididos em dois grupos: GI, pacientes que } \\
\text { receberam treinamento de força muscular } \\
\text { periférica (TMP) e treinamento de força } \\
\text { muscular respiratório (TMR) associados à } \\
\text { fisioterapia convencional, uma sessão por } \\
\text { dia para } 5 \text { dias; e GC, pacientes que } \\
\text { receberam fisioterapia convencional, uma } \\
\text { sessão por dia durante } 5 \text { dias. Foram } \\
\text { utilizados instrumentos como } \\
\text { manovacuometria, dinamometria, TC6min e } \\
\text { questionário de saúde de } 36 \text { itens. }\end{array}$ & $\begin{array}{l}\text { Ambos os grupos mostraram uma redução } \\
\text { significativa da força muscular respiratória } \\
\text { após a CRM; no entanto, no GI, a força } \\
\text { muscular inspiratória a redução foi menor. } \\
\text { A qualidade de domínios vivos para dor e } \\
\text { percepção de vitalidade piorou no controle e } \\
\text { melhorou no GI. Ambos os grupos } \\
\text { apresentaram redução da capacidade } \\
\text { funcional e da força muscular expiratória } \\
\text { após a CRM. }\end{array}$ \\
\hline $\begin{array}{c}\text { Heart, Lung and } \\
\text { Circulation }\end{array}$ & $\begin{array}{l}\text { Eficácia da espirometria de } \\
\text { incentivo em força muscular } \\
\text { inspiratória após cirurgia de }\end{array}$ & $\begin{array}{l}\text { Ensaio clínico randomizado realizado com } \\
90 \text { pacientes, com } 47 \text { e } 43 \text { pacientes } \\
\text { atribuídos aos GI e GC, respectivamente. }\end{array}$ & $\begin{array}{l}\text { Em ambos os grupos, houve uma redução } \\
\text { significativa na PImáx da linha de base pré- } \\
\text { operatória para }\end{array}$ \\
\hline
\end{tabular}




\begin{tabular}{|c|c|c|c|}
\hline $\begin{array}{l}\text { Manapunsopee S, et al. } \\
\text { (2019) }\end{array}$ & $\begin{array}{l}\text { enxerto de bypass da artéria } \\
\text { coronária. }\end{array}$ & $\begin{array}{l}\text { Programado para passar por CRM, o GI } \\
\text { recebeu espirometria de incentivo e com } \\
\text { exercício de respiração profunda (ERP), e o } \\
\text { GC recebeu apenas ERP. Pressão } \\
\text { inspiratória máxima (PImáx) antes da } \\
\text { cirurgia e no dia } 4 \text { após a cirurgia foi } \\
\text { avaliada por um medidor de pressão } \\
\text { respiratória. }\end{array}$ & $\begin{array}{l}4^{\circ} \text { dia de pós-operatório; no entanto, a } \\
\text { PImáx no grupo de espirometria de } \\
\text { incentivo teve um valor significativamente } \\
\text { menor redução na PImáx em comparação } \\
\text { com o grupo de controle. }\end{array}$ \\
\hline $\begin{array}{l}\text { Research article } \\
\text { Ribeiro BC et al. } \\
\text { (2020) }\end{array}$ & $\begin{array}{l}\text { Diferentes protocolos de } \\
\text { fisioterapia após cirurgia de } \\
\text { revascularização do miocárdio: } \\
\text { um ensaio clínico } \\
\text { randomizado. }\end{array}$ & $\begin{array}{l}\text { Ensaio controlado randomizado com } \\
\text { alocação e cegamento do pesquisador e } \\
\text { análise de intenção de tratar } 48 \text { pacientes } \\
\text { submetidos à revascularização do miocárdio } \\
\text { foram aleatoriamente } \\
\text { atribuído a um GC, grupo de mobilização } \\
\text { precoce (GMP) ou realidade virtual } \\
\text { grupo (GRV). GC realizou fisioterapia } \\
\text { respiratória e exercícios metabólicos, o GMP } \\
\text { realizou exercícios e deambulação em ciclo } \\
\text { ergômetro, e o GRV realizou as mesmas } \\
\text { atividades do GMP, com a adição de dois } \\
\text { jogos Nintendo Wii durante } 3 \text { dias de pós- } \\
\text { operatório. Foi analisado o quarto dia de } \\
\text { pós-operatório e o tempo de alta hospitalar. }\end{array}$ & $\begin{array}{l}\text { O GRV apresentou menor tempo de } \\
\text { internação. O GC mostrou um declínio na } \\
\text { variabilidade da frequência cardíaca (VFC) } \\
\text { do período pré-operatório ao quarto dia pós- } \\
\text { operatório. GMP e GRV apresentaram } \\
\text { maior modulação autonômica cardíaca em } \\
\text { comparação com o GC ( } p<0,05) \text {, com } \\
\text { melhora atividade parassimpática. }\end{array}$ \\
\hline $\begin{array}{c}\text { Journal of } \\
\text { Cardiopulmonary } \\
\text { Rehabilitation and } \\
\text { Prevention }\end{array}$ & $\begin{array}{l}\text { Efeito do treinamento muscular } \\
\text { inspiratório pré-operatório nos } \\
\text { gradientes de oxigênio alvéolo- } \\
\text { arterial após cirurgia de } \\
\text { revascularização do miocárdio }\end{array}$ & $\begin{array}{l}\text { Os pacientes foram aleatoriamente } \\
\text { designados para o GI }(\mathrm{n}=20) \text { ou o } \mathrm{GC}(\mathrm{n}= \\
\text { 20). O GI recebeu TMI pré-operatório } \\
\text { treinamento com_carga linear de pressão e } \\
\text { educação seguida de TMI pós-operatório e } \\
\text { mobilização precoce. O GC recebeu } \\
\text { educação pré-operatória e cuidados de } \\
\text { fisioterapia de rotina no pós-operatório. }\end{array}$ & $\begin{array}{l}\text { O TMI pré e pós-operatório resultaram em } \\
\text { melhora da potência da musculatura } \\
\text { inspiratória, do gradiente alvéolo-arterial e } \\
\text { da saturação de oxigênio. }\end{array}$ \\
\hline
\end{tabular}

Fonte: Autores.

\section{Discussão}

Os estudos evidenciaram que as técnicas de fisioterapia respiratória são muito importante principalmente quando elas são utilizadas antes e após a cirurgia cardíaca, pois através de atividades físicas aeróbicas contribuem para que não ocorram complicações na recuperação, diminuem o quadro álgico e melhoram a qualidade de vida, apresentando eficácia também em outros eventos cardiovasculares como o infarto, promovendo alterações hemodinâmicas e diminuindo o número de intercorrências clinicas, favorecendo a diminuição de internações e como consequência, menos gastos aos sistemas de saúde (Moreira et al., 2019).

Buscando compreende os benefícios da fisioterapia respiratória em pacientes antes de procedimento cirúrgico cardiológico, Steinmetz et al. (2020), avaliou o uso das técnicas em paciente no pré-operatório e os comparou com os pacientes eletivos de CRM, no período de duas semanas. Foram utilizados teste de exercício cardiopulmonar, teste de caminhada de seis minutos (TC6min), cronometrado - Teste Up-and-Go (TUG) e questionário de qualidade de vida. E os resultados apontaram que a reabilitação cardíaca no pré-operatório contribui para a melhora da capacidade funcional, capacidade de exercícios e qualidade de vida trazendo benefícios não só no pré-operatório como também contribui para resultados positivos no pósoperatório.

Cseka et al. (2019) reforça para os cuidados pré-operatórios, pois pacientes que recebem fisioterapia apenas após a cirurgia, tem a probabilidade grande de ter lesões pulmonares. Quando a fisioterapia é realizada também antes, pode prevenir complicações nos pulmões e caso aconteça alguma situação de risco no pós-cirúrgico, tem como repará-los. De acordo com o autor, a mobilização precoce é considerada um comportamento terapêutico que pode promover mudanças fisiológicas como 
melhorar a circulação, metabolismo, ventilação, perfusão central e periférico, além de adicionar status de prontidão para os músculos. Isso é benéfico para pacientes adultos, inclusive crianças cardiopatas congênitas.

Com o mesmo intuito de compreender melhor a importância da fisioterapia pré-operatória, mas como também pós, Manapunsopee et al. (2019) realizou um ensaio clínico com pacientes agendados para CRM. Ele dividiu o estudo em dois grupos: um grupo recebeu espirometria de incentivo e treino de expiração profunda e o outro grupo, controle, teve apenas exercício de respiração profunda. Em ambos os grupos, houve uma redução significativa na PImáx, da linha de base préoperatória e se estendendo para quarto dia a mais no pós-operatório. No entanto, a PImáx no grupo de espirometria de incentivo, teve uma redução no valor da PImáx, o que não aconteceu com o grupo de controle. Além disso, os grupos de estudo tiveram recuperação melhor da força muscular inspiratória no quarto dia de pós-CRM do que os pacientes do grupo controle.

Cordeiro et al. (2021), na mesma linha de raciocínio de Manapunsopee et al. (2019) realizou uma pesquisa para saber a importância da fisioterapia, porém pós-CRM. O estudo foi realizado com 56 pacientes de CRM, dividindo em três grupos de análise: controle (GC), grupo de treinamento com carga linear de pressão (TMI) e grupo de incentivo inspiratório (GI). Concluiu-se que houve uma redução significativa em todas as variáveis em relação à PImax. O grupo TMI obteve um valor maior na PImax e na PEmax no pós-operatório. Em relação ao Teste de Caminhada de seis minutos (TC6), houve menor perda no TMI, concluindo que o treinamento muscular com dispositivo de carga pressórica linear é superior ao treinamento com incentivo inspiratório na capacidade funcional e de força muscular em pacientes submetidos a CRM.

Por isso que de acordo Barbosa et al. (2018), o pós-CRM é um período crítico pois a cirurgia prejudica a mecânica pulmonar e leva a uma diminuição do volume dos pulmões, com redução da complacência pulmonar e aumento do trabalho, causando uma dificuldade em respirar devido à dor do tubo de drenagem que é colocado e o repouso no leito, no qual o paciente está mais propenso a desenvolver atelectasia, como também, uma possível infecção pulmonar.

Isso porque, os pacientes submetidos à cirurgia de revascularização do miocárdio na maioria das vezes desenvolvem disfunção pulmonar pós-operatória com redução significativa no volume pulmonar, mecânica respiratória prejudicada, complacência pulmonar diminuída e aumento do trabalho respiratório, e podem sentir fraqueza física, como fadiga com caminhadas ou exercícios prolongados. Assim, os principais objetivos da fisioterapia no pós-operatório de cirurgia cardíaca são a reabilitação da função cardiopulmonar, prevenindo complicações e morbidade, garantindo melhor recuperação da função pulmonar e a restauração da qualidade de vida desses pacientes (Ghiotto, 2021).

Assim, Windmöller et al. (2020), em seu estudo, buscou avaliar os tipos de intervenção fisioterapêutica usadas no pósoperatório de pacientes submetidos a cirurgia cardíaca. Eles observaram que tanto o protocolo de exercício padronizado, como o protocolo de treinamento físico com cicloergômetro associado com Pressão Positiva Contínua nas Vias Aéreas (CPAP), contribuiu para que houvesse um menor tempo de internação em Unidade de Terapia Intensiva (UTI), além de melhorar a manutenção da capacidade funcional do paciente.

Uma Reabilitação Cardiovascular (RC) é uma estratégia de tratamento fisioterapêutico juntamente complementar ao medicamento e que precisa ser pensada nas particularidades de cada paciente. A RC consiste em atividades de cinesioterapêuticos, aeróbio e resistente, sendo eles divididos em fases importantes. O progresso de uma fase para outra é executada diante à estabilidade clínica do paciente durante a cada sessão, como também, a melhoria de seus parâmetros e eficiência funcional (Durmuş et al., 2021).

No estudo de Ribeiro et al. (2020), realizado com 48-paciente submetidos a CRM, foram criados três grupos, grupo controle (CG), grupo de mobilização precoce (GMP) e grupo de realidade virtual (GRV). O GC realizou fisioterapia respiratória e exercícios metabólicos; o GMP realizou exercícios em cicloergômetro e deambulação; e o GRV realizou as mesmas atividades do GMP, com adição de dois jogos virtuais durante três dias de pós-operatório, sendo analisadas as variáveis de variabilidade da frequência cardíaca no pré-operatório, quarto dia de pós-operatório e tempo de alta hospitalar. 
Logo, o GRV apresentou menor tempo de internação, o GC apresentou um declínio na variação da frequência cardíaca do período pré-operatório ao quarto dia pós-operatório. O GMP e o GRV apresentaram maior modulação autonômica cardíaca em relação ao GC, com melhora da atividade parassimpática, concluindo que a variação de diferentes protocolos de intervenção fisioterapêutica afetou a modulação autonômica da frequência cardíaca e o tempo de internação em pacientes submetidos à CRM.

Segundo Bourscheid et al. (2021), um exercício aeróbio é importante pós-procedimento cirúrgico pois aumenta a diferença arteriovenosa de oxigênio, que está diretamente relacionada ao aumento do VO2pico, por meio de uma maior oferta de oxigênio periférico, graças à produção de catecolaminas e maior biodisponibilidade de óxido nítrico. A função cardíaca também funciona melhor, pois há um aumento do enchimento diastólico, ao mesmo tempo em que combina pré-carga aumentada, causando um relaxamento miocárdico ideal. Melhorar a função cardíaca pós-CRM trará benefícios significativos em termos de menor tempo diastólico e maior eficiência do enchimento dele.

Logo, Aquino et al. (2021), confirma essa mesma teoria, quando pesquisou a eficiência do treinamento muscular respiratório e periférico em pacientes submetidos à CRM. Como resultado do seu estudo, afirmou que o treinamento muscular respiratório e periférico associado à fisioterapia convencional, sendo realizando uma sessão por dia durante cinco dias, trouxe maiores benefícios do que somente a fisioterapia convencional durante cinco dias corrido, em período de pós-operatório em pacientes submetidos à CRM. Os exercícios podem reduzir a perda da força muscular inspiratória, melhora da percepção da dor e vitalidade imediato pós-CRM.

Nesse sentido, Costa et al. (2021) adverte que, as atividades físicas nesses pacientes muitas vezes são reduzidas, causando fadiga, falta de ar e fraqueza da musculatura inspiratória, limitando o exercício, piorando a qualidade de vida a cada dia que passa, além de quê desencadeia um ciclo vicioso com efeitos psicológicos negativos, contribuindo para a exacerbação dos sintomas e intolerância ao exercício, resultando em redução da capacidade funcional. Por tanto, sugere uma orientação sobre a importância dos exercícios, um pré-condicionamento antes da cirurgia e uma constância nos exercícios pós-CRM, sempre respeitando os limites funcionais de cada paciente e dando oportunidades para aqueles que estão enfraquecidos e em estado grave.

Turky (2017), em seu estudo, buscou intervir com atividades físicas e orientação pré-cirurgia e pós sobre a importância da fisioterapia. A intervenção aconteceu com um grupo de 20 pacientes que recebeu TMI e mobilização precoce no pós-operatório de CRM, já o grupo controle, recebeu educação pré-operatória e cuidados de fisioterapia de rotina no pósoperatório. O resultado foi que, o grupo de intervenção mostrou melhora significativa dos gradientes alveolares de oxigênio arterial e potência do músculo inspiratório e em todos os pontos de medição. A alta na potência média do músculo inspiratório foi significativamente maior do que na linha de base, mas não estatisticamente significativamente diferente da medida préoperatória.

Corroborando com Turky (2017), o estudo de Sahar et al. (2020), afirma que a eficácia do treinamento muscular respiratório versus o tratamento convencional, de acordo com as leituras pré e pós-operatórias, mostraram que os pacientes tem um maior desempenho, apresentando menor tempo de ventilação mecânica, dependência de oxigenoterapia e internação pósoperatória em relação ao grupo controle, apresentando valores de $p$ abaixo de 0,05 , mostraram uma melhora na capacidade funcional pós operatório e redução do tempo de internação hospitalar.

Silva et al. (2021) completam dizendo, que as técnicas de respiração não invasivas são necessárias durante a internação hospitalar, tanto no período pré e no pós-operatório para prevenir efeitos de tempo no leito, otimização a independência funcional, redução das complicações pulmonares por meio da manutenção do trato respiratório permeável, evitando o uso de ventilação mecânica invasiva (VMI) e extubação. 
Independente de atividade, qualquer incentivo de treinamento dos músculos respiratórios, melhora a força dos músculos respiratórios e reduz sintomas, como falta de ar e intolerância ao exercício e uma boa recuperação desses pacientes, principalmente após o uso de VM (Dias et al., 2021).

Em uma última analise, no estudo de Sinnadurai et al. (2020), foi evidenciado os benefícios no processo de reabilitação cardiovascular. A pesquisa que teve como objetivo comparar os efeitos da reabilitação cardíaca (RC) entre pacientes que realizaram e os que não realizaram após 18 meses da CRM. Concluiram que a RC contribui de forma significativa para o controle dos fatores de risco em pacientes com doença isquêmica do coração, comparando os resultados dos pacientes que participaram em relação àqueles que não realizaram a $\mathrm{RC}$, pois os pacientes que participaram eram mais propensos a parar de fumar, alcançando controle de glicose aceitável e melhor qualidade de vida, porém são necessários esforços adicionais para aumentar os encaminhamentos para RC em pacientes, devendo ser acompanhados por uma estratégia de longo prazo para manter os efeitos benéficos.

Nesse sentido, Soares, Sousa e Medeiros (2017) reforçam, que o que todos os autores conseguiram descobrir, que a fisioterapia respiratória é extremamente importante na prevenção e controle de complicações pulmonares após cirurgia cardíaca e deve ser iniciado o mais rápido possível. Entre as técnicas utilizadas antes da cirurgia, destacam o treinamento do padrão diafragmático, o redirecionamento do fluxo aéreo, os exercícios inalatórios e a reexpansão pulmonar. Em relação ao pós-operatório de cirurgia cardíaca, podem ser citadas técnicas de drenagem postural, exercícios de respiração profunda, estimulação da tosse, compressões torácicas e hiperinsuflação manual.

\section{Conclusão}

Observou-se por meio das pesquisas em bases cientificas, que as intervenções fisioterapêuticas aplicadas ao período de pré e pós-cirúrgico de revascularização do miocárdio sugerem que as diretrizes associadas a exercícios respiratórios devem ser aplicadas antes da cirúrgica e pós-operatórias, encurtando a duração da permanência em pacientes submetidos à cirurgia de RM. Além disso, contribui para o ganho funcional e melhoria da qualidade de vida dos pacientes cardíacos, evidenciando a importância da presença do fisioterapeuta na equipe multiprofissional nos hospitais que realizam CRM, ao mesmo tempo que mostrou que a fisioterapia pré e pós-operatória provou ter ferramentas que podem auxiliar os fisioterapeutas na reabilitação de pacientes, facilitando sua recuperação e devendo ser uma prática adaptada às características da população atendida.

É importante relatar a necessidade de pesquisa que evidenciem a fisioterapia pré e pós CRM para a prevenção e reabilitação de pacientes afim de reduzir o risco operacional e melhorar resultados de curto e longo prazo após a cirurgia. Ademais, sugere-se, o desenvolvimento de estudos futuros em linhas de pesquisas que incluam trabalhos sobre as intervenções fisioterapêuticas no pré-operatório de cirurgia de revascularização do miocárdio e sobre a fisioterapia de expansão de função respiratória, visto que foi observada uma carência de estudos nesse sentido.

\section{Referências}

Aquino, T. N., Rosseto, S.F, Vaz, J.L, Alves, C. F. C, Vidigal, F. D. C., \& Galdino, G. (2021). Evaluation of respiratory and peripheral muscle training in individuals undergoing myocardial revascularization. Journal of Cardiac Surgery. 36(9). https://doi.org/10.1111/jocs.15698

Araújo, T. M., Junior, M. A. D. V. C., Carvalho, S. O., \& Peruna, M. P. (2016). Tempo de ventilação mecânica e força muscular periférica na pós-cirurgia cardíaca. International Journal of Cardiovascular Sciences, 29(2), 134-138. 10.5935/2359-4802.20160021

Barbosa, J. L., Thiers, C. A., Cunha, C. F. D. S., Moutella, J., Tura, B. R., Orsi, G. P., ... \& Faria, L. F. (2018). Impacto dos Fatores de Risco para Doença Arterial Coronariana nos Gastos Hospitalares dos Pacientes Submetidos à Cirurgia de Revascularização do Miocárdio no SUS. International Journal of Cardiovascular Sciences, 31, 90-96. https://doi.org/10.5935/2359-4802.20170098

Bourscheid, G., Just, K. R., Costa, R. R., Petry, T., Danzmann, L. C., Pereira, A. H., \& Garcia, E. L. (2021). Efeito de diferentes modalidades de treinamento físico no consumo de oxigênio de pico em pacientes pós-infarto agudo do miocárdio: uma revisão sistemática e metanálise. Jornal Vascular Brasileiro, 20. https://doi.org/10.1590/1677-5449.210056 
Cavenaghi, S. et al. (2011). Fisioterapia respiratória no pré e pós-operatório de cirurgia de revascularização do miocárdio. Brazilian Journal of Cardiovascular Surgery, 26(1), 455-461. https://doi.org/10.5935/1678-9741.20110022

Cordeiro, A. L. L., Soares, L. O., Vasconcelos, M. L. L., da Paixão Correia, T. R., de Souza, A. S., Guimarães, A. R. F., \& de Andrade, P. H. C. (2021). Dois tipos de treinamento muscular inspiratório sobre a força muscular de pacientes após revascularização do miocárdio: ensaio clínico. Fisioterapia Brasil, 22(3), 290-305. https://doi.org/10.33233/fb.v22i3.4796

Costa, S. A. S., Epaminondas, L. C. S., Pena, J. C. V., Lima, P. T. S., Negrão, L. N., Sassim, P. VS., \& Ferreira, T. C. R. (2020). A atuação da fisioterapia no pós-operatório de transplante de coração: uma revisão da literatura. Revista cpaqv, 12(3), 2. 10.36692/v12n3-26r

Costa, L. S., de Azevedo, L. P. C., \& Martins, I. C. (2021). Programa de reabilitação cardíaca associado ao treinamento muscular inspiratório e na capacidade funcional em pacientes com insuficiência cardíaca: uma revisão. Revista Ibero-Americana de Humanidades, Ciências e Educação, 7(2), 15-15. https://doi.org/10.51891/rease.v7i2.632

Cseka, B.L. A et al. (2019). Fisioterapia no pré e pós-operatório de cirurgia cardíaca pediátrica: revisão bibliográfica. Revista Ciência e Saúde On-line 4(1),1723. https://revistaeletronicafunvic.org/index.php/c14ffd10/article/view/119/113

Durmuş, İ., Kalaycıŏlu, E., Çetin, M., Şahin, H. B., \& Kırış, T. (2021). Reabilitação Cardíaca Baseada em Exercícios Fortemente Relacionada com Redução do Volume Plaquetário Médio. Arquivos Brasileiros de Cardiologia. https://doi.org/10.36660/abc.20190514

Ghiotto, T. B., \& Dias, T. Z. G. (2021). A atuação da fisioterapia pós-cirurgia cardíaca: revisão de literatura. Anais do EVINCI-UniBrasil, 7(1), 414-414. https://portaldeperiodicos.unibrasil.com.br/index.php/anaisevinci/article/view/6225

Leguisamo, C.P, Kalil, R.A.K \& Furlani, A.P. (2005). A efetividade de uma proposta fisioterapêutica pré-operatória para cirurgia de revascularização do miocárdio. Brazilian Journal of Cardiovascular Surgery, 20(1), 134-141. https://www.scielo.br/j/rbccv/a/kwdhckkRT9rPDQp5fpSbLzv/?format=pdf\&lang=pt

Manapunsopee, S., Thanakiatpinyo, T., Wongkornrat, W., Chuaychoo, B., \& Thirapatarapong, W. (2020). Effectiveness of incentive spirometry on inspiratory muscle strength after coronary artery bypass graft surgery. Heart, Lung and Circulation, 29(8), 1180-1186. https://doi.org/10.1016/j.hlc.2019.09.009

Mota, É. C., Oliveira, S. P., Silveira, B. R., Silva, P. L., \& Oliveira, A. C. (2017). Incidência da pneumonia associada à ventilação mecânica em unidade de terapia intensiva. Medicina (Ribeirão Preto) 50(1), 39-46. https://doi.org/10.11606/issn.2176-7262.v50i1p39-46

Moreira, W. E. M, \& Cassimiro, M. S. (2019). O papel do fisioterapeuta respiratório na abordagem do paciente com insuficiência respiratória: realidades da assistência domiciliar. Revista Saúde e Desenvolvimento, 13(15), 134-142. https://www.revistasuninter.com/revistasaude/index.php/as udeDesenvolvimento/article/view/920

Osório, A. C. M., Flores, L., Marin, L., Zanini, S. C. C., \& Leguisamo, C. P. (2014). Qualidade de vida de pacientes idosos submetidos à cirurgia de revascularização. Revista FisiSenectus, 2(1), 13-23. https://doi.org/10.22298/rfs.2014.v2.n1.2789

Ribeiro, B. C., Poça, J. J. G. D., Rocha, A. M. C., Cunha, C. N. S. D., Cunha, K. D. C., Falcão, L. F. M., \& Rocha, R. S. B. (2021). Different physiotherapy protocols after coronary artery bypass graft surgery: A randomized controlled trial. Physiotherapy Research International, 26(1), e1882. https://doi.org/10.1002/pri.1882

Sá, P. C., Lopes, N. L. S., Costa, N. L., da Silva, M. L., do Nascimento, R. G., \& da Costa Cunha, K. (2020). Avaliação da atividade física de pacientes no préoperatório de cirurgia cardíaca. Saúde (Santa Maria), 46(2). https://doi.org/10.5902/2236583443823

Sahar, W., Ajaz, N., Haider, Z., \& Jalal, A. (2020). Effectiveness of Pre-operative Respiratory Muscle Training versus Conventional Treatment for Improving Post operative Pulmonary Health after Coronary Artery Bypass Grafting. Pakistan Journal of Medical Sciences, 36(6), 1216. 10.12669 / pjms.36.6.2899

Santos, J. N., de Moura, A. É. F. R., de Sousa, A. L. L., Rodrigues, I. S., de Souza Vasconcelos, L. T., Irineu, M. E. N., \& Modesto, T. S. F. C. (2019). Atuação fisioterapêutica no pós-operatório de cardiopatias congênitas pediátricas. Biomotriz, 13(1). https://www.researchgate.net/profile/Jefferson-DosSantos/publication/332544681_Atuacao_fisioterapeutica_no_posoperatorio_de_cardiopatias_congenitas_pediatricas_Physiotherapy_in_the_postoperative_of_ pediatric_congenital_cardiopathies/links/5cbb8510299bf1209774794a/Atuacao-fisioterapeutica-no-pos-operatorio-de-cardiopatias-congenitas-pediatricas-

Physiotherapy-in-the-postoperative-of-pediatric-congenital-cardiopathies.pdf

Silva, D. H., Pereira, T. M. A., \& de Moraes Silva, J. (2021). Técnicas respiratórias na melhora da função pulmonar de cardiopatas: Uma Revisão Integrativa. Revista de Saúde, 12(3), 26-32. https://doi.org/10.21727/rs.v12i3.2641

Sinnadurai, S., Sowa, P., Jankowski, P., Gąsior, Z., Kosior, D. A., Haberka, M., \& Kamiński, K. (2021). Effects of cardiac rehabilitation on risk factor management and quality of life in patients with ischemic heart disease: A multicenter cross-sectional study. Polish Archives of Internal Medicine, 131(7), 617625. 10.20452 / pamw.16019

Soares, J. L., de Sousa, A. M. B., \& da Silva Medeiros, J. (2017). Tratamento Fisioterapêutico no pré e pós-operatório de cirurgia cardíaca: revisão integrativa. Revista Ciência \& Saberes-UniFacema, 3(3), 624-629. http://www.facema.edu.br/ojs/index.php/ReOnFacema/article/view/191/134

Steinmetz, C., Bjarnason-Wehrens, B., Baumgarten, H., Walther, T., Mengden, T., \& Walther, C. (2020). Prehabilitation in patients awaiting elective coronary artery bypass graft surgery-effects on functional capacity and quality of life: a randomized controlled trial. Clinical rehabilitation, 34(10), 1256-1267. 10.20452/pamw.16019

Turky, K., \& Afify, A. M. A. (2017). Effect of Preoperative Inspiratory muscle training on alveolar-arterial oxygen gradients after coronary artery bypass surgery. Journal of cardiopulmonary rehabilitation and prevention, 37(4), 290-294. 10.1097/HCR.0000000000000234

Windmöller, P., Bodnar, E. T., Casagrande, J., Dallazen, F., Schneider, J., Berwanger, S. A., \& Winkelmann, E. R. (2020). Physical exercise combined with CPAP in subjects who underwent surgical myocardial revascularization: a randomized clinical trial. Respiratory care, 65(2), 150-157. https://doi.org/10.4187/respcare.06919 